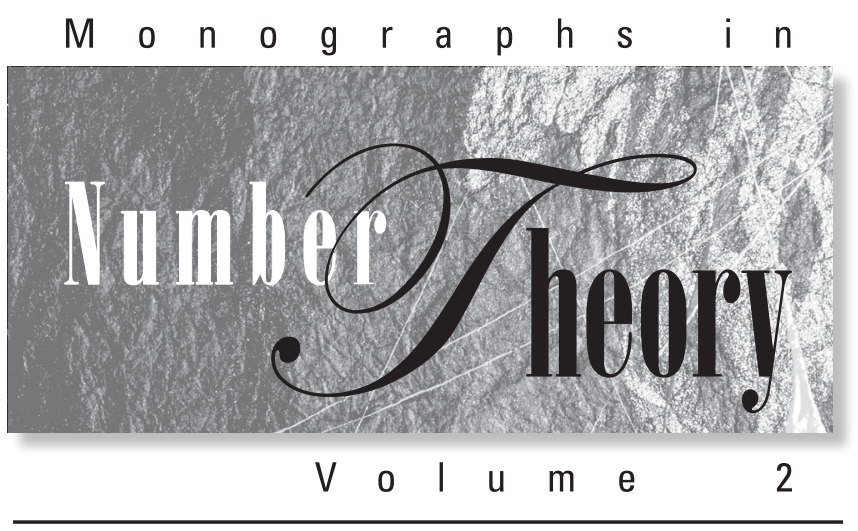

Topics in

Number Theory 
Series Editors: Bruce C. Berndt

(University of Illinois at Urbana-Champaign, USA)

Heng Huat Chan

(National University of Singapore, Singapore)

\title{
Editorial Board Members:
}

Jonathan M. Borwein (Dalhousie University, Canada; The University of Newcastle, Australia)

William Duke (University of California at Los Angeles, USA)

Wen-Ching Winnie Li (The Pennsylvania State University, USA)

Kannan Soundararajan (Stanford University, USA)

Wadim Zudilin (Steklov Mathematical Institute, Russia;

The University of Newcastle, Australia)

\author{
Published \\ Vol. 1 Analytic Number Theory - An Introductory Course \\ by Paul T. Bateman \& Harold G. Diamond \\ Vol. 2 Topics in Number Theory \\ by Minking Eie
}


$\begin{array}{llllllllllllllllll}M & 0 & n & 0 & g & r & a & p & h & s & & i & n\end{array}$

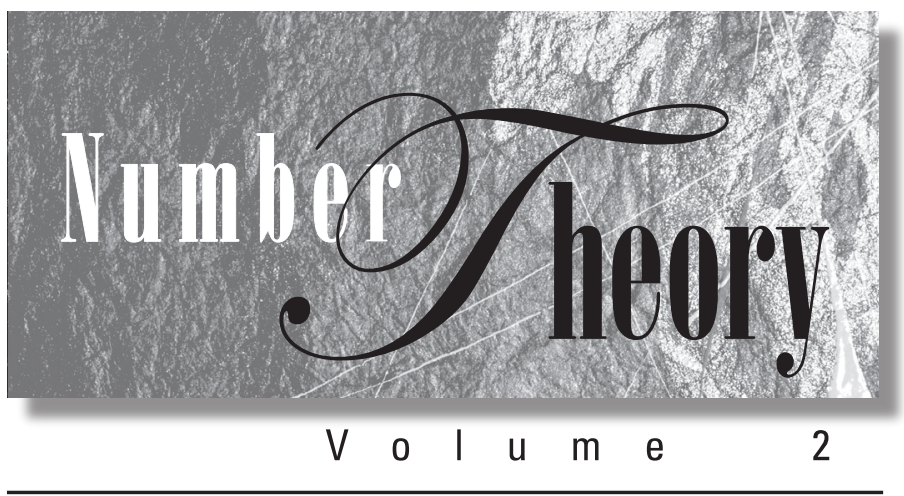

Topics in

Number Theory

\author{
Minking Eie \\ National Chung Cheng University, Taiwan
}




\section{Published by}

World Scientific Publishing Co. Pte. Ltd.

5 Toh Tuck Link, Singapore 596224

USA office: 27 Warren Street, Suite 401-402, Hackensack, NJ 07601

UK office: 57 Shelton Street, Covent Garden, London WC2H 9HE

\section{British Library Cataloguing-in-Publication Data}

A catalogue record for this book is available from the British Library.

\section{TOPICS IN NUMBER THEORY \\ Monographs in Number Theory - Vol. 2}

Copyright () 2009 by World Scientific Publishing Co. Pte. Ltd.

All rights reserved. This book, or parts thereof, may not be reproduced in any form or by any means, electronic or mechanical, including photocopying, recording or any information storage and retrieval system now known or to be invented, without written permission from the Publisher.

For photocopying of material in this volume, please pay a copying fee through the Copyright Clearance Center, Inc., 222 Rosewood Drive, Danvers, MA 01923, USA. In this case permission to photocopy is not required from the publisher.

ISBN-13 978-981-283-518-5

ISBN-10 981-283-518-0

Printed in Singapore. 


\section{Preface}

Since I left the Institute of Mathematics at Academia Sinica in 1991, I have conducted a graduate course on number theory every year at the National Chung Cheng University. The title of the course is usually "Special Topics in Number Theory" or "Theory of Modular Forms of Several Variables". Unfortunately, there are no textbooks available for such a course and I had to write my own lecture notes, lifting materials from books by T. Shimura [48], E. Freitag [31], W. L. Baily, Jr. [6] and T. Miyake [42]. To enrich the contents of the lecture notes, I have included some of the results from my own research work.

In such a course as mentioned above, we usually begin with the theory of modular forms of one variable, followed by the theory of zeta functions, new approaches to Bernoulli identities and Kummer Congruences, the theory of modular forms of several variables, the theory of Jacobi forms over Cayley numbers and finally the theory of Jacobi forms of several variables over Cayley numbers. The theory of Jacobi forms is based on my research papers from 1991 to 2000. Students are encouraged to conduct research related to the materials mentioned above. Eventually, a total of 6 Ph.D. students and more than 30 M.Sc. students graduated under this programme.

The theory of modular forms of several variables [31] was initiated by C. L. Siegel around 1945 in an attempt to generalize the theory of modular forms of one variable. The theory was employed by M. Eichler and D. Zagier [15] in 1985 to give a definition of Jacobi forms. Indeed the coefficients of the Fourier-Jacobi expansions of modular forms of rank two are the main sources of Jacobi forms. It is still a difficult problem to construct Jacobi forms besides the Eisenstein series. Even the Eisenstein series of Jacobi 
forms of several variables are difficult to construct. All we can say is that many results concerning modular forms of several variables remain to be studied in depth.

Georg-August-Universität Göttingen is the center of development of modular forms with masters like Siegel and U. Christian. I spent a year at Georg-August-Universität Göttingen as a Sonderforschungsbereich (special research fellow) in 1985, three years after my graduation from the University of Chicago in 1982.

It is almost 60 years since the birth of modular forms of several variables in Germany. It is an appropriate time to have a textbook on the theory in English rather than in German. This is my main motivation for writing this book.

Minking Eie April 19, 2008 


\section{Glossary}

$\mathrm{SL}_{2}(\mathbb{R}) \quad$ special linear group over $\mathbb{R} \quad 3$

$\mathscr{H} \quad$ upper half-plane of $\mathbb{C} \quad 3$

$\mathrm{SL}_{2}(\mathbb{Z}) \quad$ special linear group over $\mathbb{Z}$

$j(g, z) \quad=c z+d$ if $g=\left[\begin{array}{ll}a & b \\ c & d\end{array}\right] \in \mathrm{SL}_{2}(\mathbb{R})$

$\Gamma \quad=\mathrm{SL}_{2}(\mathbb{Z}) \quad 5$

$\bar{\Gamma} \quad=\mathrm{SL}_{2}(\mathbb{Z}) /\left\{ \pm E_{2}\right\}$, full modular group 5

$\Gamma_{0} \quad$ maximal parabolic subgroup of $\Gamma \quad 5$

$\Gamma \backslash \mathscr{H} \quad$ orbit space of $\Gamma$ acting on $\mathscr{H} \quad 6$

$\mathscr{F} \quad$ a fundamental domain of $\mathscr{H}$ with respect to $\Gamma \quad 6$

$G_{k}(z) \quad$ Eisenstein series of weight $k \quad 10$

$\mathscr{L} \quad=\{m z+n \mid m, n \in \mathbb{Z}\}$, a lattice of $\mathbb{C} \quad 10$

$\zeta(s) \quad$ Riemann zeta function 11

$\sigma_{k}(n) \quad$ divisor function 12

$B_{k} \quad$ the $k$ th Bernoulli number 12

$E_{k}(z) \quad$ normalized Eisenstein series of weight $k \quad 13$

$\Gamma(s) \quad$ gamma function 18

$\pi(x) \quad$ number of prime numbers not exceeding $x \quad 18$

$\mu(n) \quad$ Möbius function 19

$\gamma \quad$ Euler constant 20

$B_{n}(x) \quad$ the $n$th Bernoulli polynomial 25

$\zeta(s ; \delta) \quad$ Hurwitz zeta function $\quad 27$

$\mathscr{S}\left(\mathbb{R}^{n}\right) \quad$ space of rapidly decreasing function on $\mathbb{R}^{n} \quad 29$

$\begin{array}{lll}\widehat{f} & \text { Fourier transform of } f & 29\end{array}$

$\langle\cdot, \cdot\rangle \quad$ inner product $\quad 29$

$\vartheta(S, z) \quad$ theta series 34 
$Z(f, s) \quad$ zeta function attached to a modular form $f \quad 36$

$T(n) \quad$ Hecke operator 40

$R_{\lambda} \quad$ homothety operator $\quad 42$

$v_{z_{0}}(f) \quad$ least integer $n$ such that $b_{n} \neq 0 \quad 47$

$v_{\infty}(f) \quad$ least integer $n$ such that $a_{n} \neq 0 \quad 47$

$A_{k}(\Gamma) \quad$ vector space of modular forms of weight $k \quad 49$

$\Delta(z) \quad$ discriminant 50

$\eta(z) \quad$ Dedekind eta function $\quad 50$

$\Lambda(f, s) \quad=(2 \pi)^{-s} \Gamma(s) \zeta(s) \zeta(s+1) \quad 51$

$X(s) \quad=\pi^{-s / 2} \Gamma\left(\frac{s}{2}\right) \zeta(s)$

$\tau(n) \quad$ Ramanujan's tau function 53

$S_{k}(\Gamma) \quad$ vector space of cusp forms of weight $k \quad 54$

$\left\{\varphi_{n}(z)\right\}_{n=1}^{N} \quad$ an orthonormal basis for $S_{k}(\Gamma)$

$L_{k}^{1}(D) \quad$ set of measurable functions on the unit disc $\quad 55$

$f_{\Gamma}(z) \quad=\sum_{\gamma \in \Gamma} f(\gamma(z)) j(\gamma, z)^{-k} \quad 56$

$C(\gamma) \quad$ centralizer of $\gamma$ in $\mathrm{SL}_{2}(\mathbb{Z}) \quad 58$

$\{\gamma\} \quad$ conjugacy class of $\gamma$ in $\mathrm{SL}_{2}(\mathbb{Z}) \quad 58$

$J^{\ell} \quad$ linear extension of $\prod_{j=1}^{\ell} X_{j}^{\alpha_{j}} \mapsto \prod_{j=1}^{\ell} \zeta\left(-\alpha_{j}\right) \quad 67$

$\begin{array}{lll}\zeta_{p}(s) \quad \text { local Riemann zeta function } & 78\end{array}$

$S_{p, q} \quad$ double Euler sum $\quad 85$

$\eta(s ; x) \quad$ Lerch zeta function $\quad 96$

$E_{n}(x) \quad$ the $n$th Euler polynomial $\quad 96$

$\psi(x) \quad$ digamma function 101

$\zeta\left(\alpha_{1}, \ldots, \alpha_{r}\right) \quad$ multiple zeta value or $r$-fold Euler sum 107

$G_{n}(p, q) \quad$ Euler sum with two branches 112

$\begin{array}{lll}M_{n}(\mathbb{R}) \quad \text { matrix ring over real numbers field of size } n & 131\end{array}$

${ }^{t} X \quad$ transpose of $X \quad 131$

$\mathcal{H}_{n} \quad$ generalized (Siegel) upper half-plane 131

$\begin{array}{lll}p_{B} & \text { a translation of } \mathcal{H}_{n} & 133\end{array}$

$\mathrm{GL}_{n}(\mathbb{R}) \quad$ general linear group over $\mathbb{R}$ of size $n \quad 133$

$\begin{array}{lll}t_{U} & \text { a dilation of } \mathcal{H}_{n} & 133\end{array}$

$\iota \quad$ inversion of $\mathcal{H}_{n} \quad 133$

$\operatorname{Sp}(n, \mathbb{R}) \quad$ real symplectic group of degree $n$ over $\mathbb{R} \quad 133$

$U(n) \quad$ unitary group of size $n \quad 139$

$\begin{array}{lll}D_{n} & \text { generalized disc } & 140\end{array}$ 
$\begin{array}{lll}M_{n}(\mathbb{C}) & \text { matrix ring over } \mathbb{C} \text { of size } n\end{array}$

$\mathfrak{H}_{n}$

Hermitian upper half-plane

142

$\mathbb{H}$

skew field of quaternions over $\mathbb{R}$

142

$\Omega_{n}$

a tube domain contained in $\mathbb{C}^{2 n^{2}+n}$

142

$\Gamma_{n}$

$=\operatorname{Sp}(n, \mathbb{Z})$

$\Gamma_{n}^{0}$

$=\left\{\left[\begin{array}{ll}A & B \\ C & D\end{array}\right] \in \Gamma_{n} \mid C=0\right\}$

145

$\Gamma_{n}(N)$

principal congruence subgroup of level $N$ of $\Gamma_{n}$

147

$\Lambda_{n}^{*}$ set of $n \times n$ half-integral symmetric matrices

151

$\sigma(X)$

$\Lambda_{n}$ trace of a matrix $X$

151

set of $n \times n$ integral symmetric matrices

151

$j(M, Z)$

$=\operatorname{det}(C Z+D)$ if $M=\left[\begin{array}{cc}A & B \\ C & D\end{array}\right] \in \operatorname{Sp}(n, \mathbb{R})$

152

$E_{k}(Z)$

Eisenstein series

152

$\gamma(s)$

$=\prod_{\nu=0}^{n-1} \Gamma\left(s-\frac{\nu}{2}\right)$

157

$F_{k}(Z)$

$=\sum_{\operatorname{det} C \neq 0} \operatorname{det}(C Z+D)^{-k}$

158

$\nu(R)$

content of $R$

158

$S[X]$

$A(S, T)$

$={ }^{t} X S X$

160

number of solutions to $S[X]=T$

160

$A_{q}(S, T)$

number of solutions to $S[X] \equiv T(\bmod q)$

160

$d_{p}(S, T)$

local density at the place $p$

161

$A_{\infty}(S, T)$

$=\lim _{N \rightarrow T} \operatorname{vol}\left(\varphi^{-1}(N)\right) / \operatorname{vol}(N)$

161

$h$

number of classes in the genus of a matrix $S$

162

$\mu(S)$

$=\sum_{i=1}^{h} w\left(S_{i}\right)^{-1}$

162

$w\left(S_{i}\right)$

$\vartheta(S, Z)$

order of the group of units of $S_{i}$

162

theta series on the Siegel upper half-plane $\quad 162$

$(A, B)$

$A_{4}(n)$

trace of $A B$

162

number of integral solutions to $\sum_{k=1}^{4} x_{k}^{2}=n \quad 166$

$\left(\frac{v}{p}\right)$

Legendre symbol

166

$\mathrm{SL}_{2}(\mathbb{Z}) \ltimes \mathbb{Z}^{2} \quad$ Jacobi group

171

$\mathfrak{f}$

a field

174

$\mathscr{C}_{\mathfrak{f}}$

Cayley numbers over $\mathfrak{f}$

174

$\mathcal{O}$

integral Cayley numbers, $\mathbb{Z}$-module in $\mathscr{C}_{\mathbb{Q}}$

174

$\mathscr{C}_{\mathbb{Q}}$

$\mathscr{H} \times \mathscr{C}_{\mathbb{C}}$ set of rational Cayley numbers

174

product space of $\mathscr{H}$ and $\mathscr{C}_{\mathbb{C}} \quad 177$

$\vartheta_{m, q}(z, w)$

theta series

179

$\Lambda(q)$

$=\{\lambda+q / m \mid \lambda \in \mathcal{O}\}$

179 


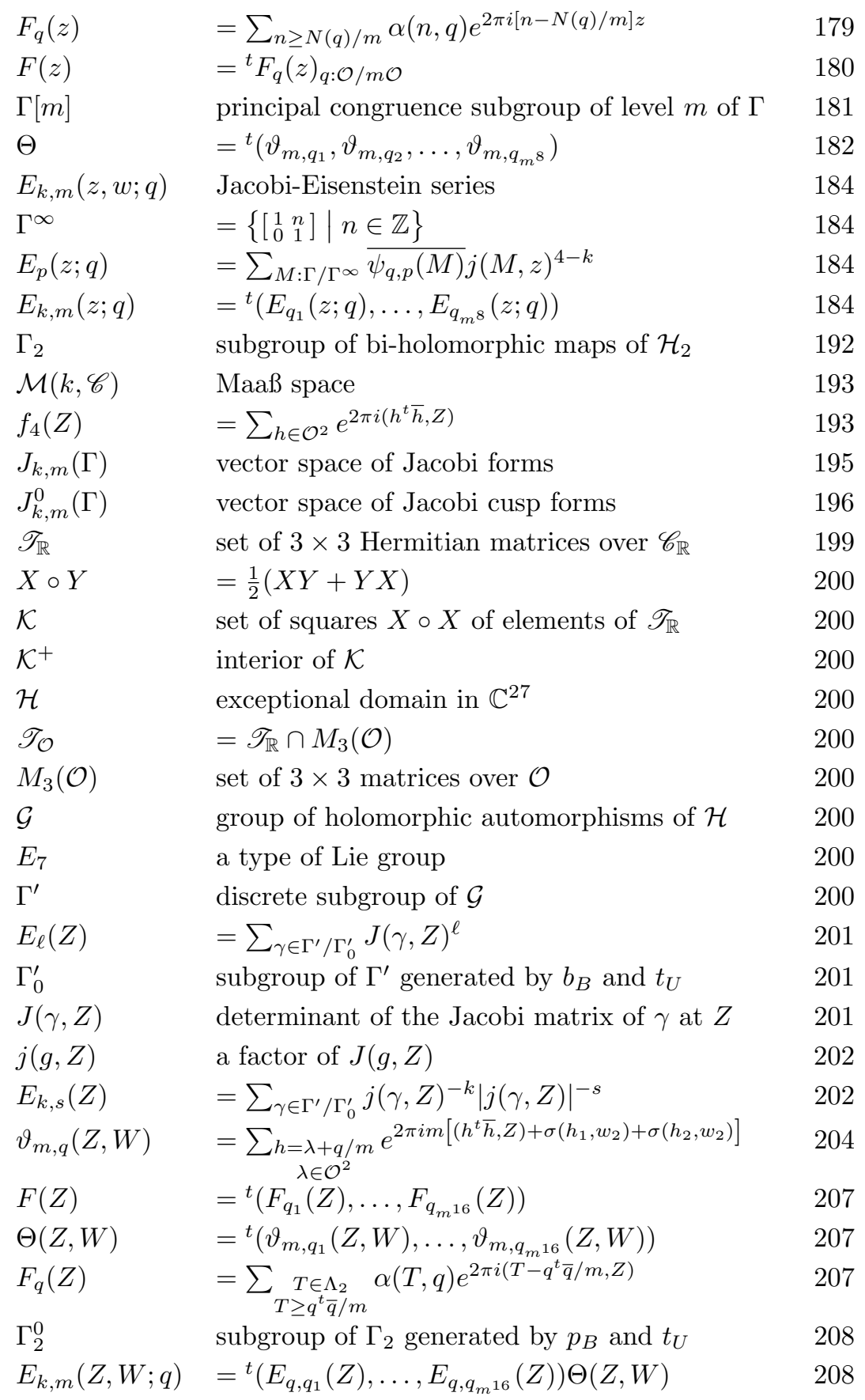




$\begin{array}{lll}E_{q, p}(Z) & =\sum_{M: \Gamma_{2} / \Gamma_{2}^{0}} j(M, Z)^{4-k} \overline{\psi_{q, p}(M)} & 208 \\ E_{4}(Z) & \text { modular form of weight } 4 \text { on } \mathcal{H} & 209 \\ E(Z) & =f_{4}(Z)+\sum_{m=1}^{\infty} \varphi_{m}\left(Z_{1}, W\right) e^{2 \pi i m z_{3}} & 210 \\ E^{2}(Z) & =\sum_{T \in \mathscr{T}_{\mathcal{O}} \cap \mathcal{K}^{+}} b(T) e^{2 \pi i(T, Z)} & 211 \\ \Gamma_{2} \backslash \mathcal{H}_{2} & \text { a fundamental domain of } \mathcal{H}_{2} \text { for } \Gamma_{2} & 213 \\ d X d Y & \text { Euclidean measure on } \mathcal{H}_{2} & 213 \\ \nu_{p}(m) & p \text {-adic valuation } & 216 \\ J_{k, m}^{0}\left(\Gamma_{2}\right) & \text { vector space of Jacobi cusp form } & 216 \\ \mathbb{Z}_{p} & \text { ring of } p \text {-adic integers } & 219 \\ \mathbb{Q}_{p} & \text { field of } p \text {-adic numbers } & 226 \\ \mu(x) & p \text {-adic measure } & 233\end{array}$


This page intentionally left blank 


\section{Contents}

Preface v v

Glossary vii

I Theory of Modular Forms of One Variable 1

1 Group Actions of the Modular Group 3

1.1 The Upper Half-Plane . . . . . . . . . . . . . . . . . . . . . . . . . . . . . .

1.2 The Modular Group . . . . . . . . . . . . . . 5

1.3 Modular Forms . . . . . . . . . . . . . . . . . . . . . 9 9

1.4 Exercises . . . . . . . . . . . . . . . . . 14

2 The Gamma and Zeta Functions $\quad 17$

2.1 The Riemann Zeta Function and the Gamma Function . . . 17

2.2 Analytic Continuation . . . . . . . . . . . . . . . . . . . . . . 21

2.3 Bernoulli Numbers . . . . . . . . . . . . . . . . . . 23

2.4 Functional Equation of $\zeta(s) \ldots \ldots \ldots \ldots$

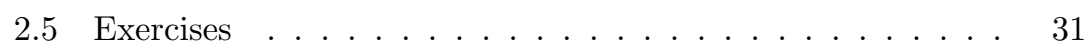

3 Zeta Functions of Modular Forms 33

3.1 Theta Series as Examples of Modular Forms . . . . . . . . 33

3.2 Zeta Functions Attached to Modular Forms . . . . . . . . . 36

3.3 Hecke Operators on Modular Forms . . . . . . . . . . . 40

3.4 Exercises ............................ 44 
4 Dimension Formulæ $\quad 47$

4.1 Dimension Formulæ for Modular Forms . . . . . . . . . . . 47

4.2 Dedekind Eta Function . . . . . . . . . . . . . 50

4.3 Selberg Trace Formula . . . . . . . . . . . . . . . . . 54

4.4 Exercises ...................... 59

5 Bernoulli Identities and Applications $\quad \mathbf{6 1}$

5.1 Two Classical Bernoulli Identities . . . . . . . . . . . . 61

5.2 Zeta Functions Associated with Linear Forms . . . . . . . . 66

5.3 Zeta Functions Associated with Rational Functions . . . . . 70

5.4 Kummer's Congruences . . . . . . . . . . . . . . 77

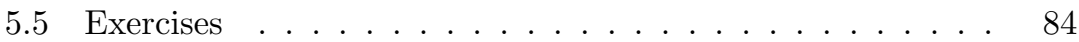

6 Euler Sums and Recent Development 85

6.1 Two Different Approaches for Evaluations . . . . . . . . . . 85

6.2 Analogue of Euler Sums . . . . . . . . . . . . . . . 93

6.2.1 Extended Euler sums [13] . . . . . . . . . . . . . 93

6.2.2 Generalized Euler sums of even weight [26] . . . . . 96

6.2.3 Euler sums over rationally deformed simplices [28] . 98

6.3 Euler Sums on Hurwitz Zeta Functions . . . . . . . . . . . . 99

6.4 Multiple Zeta Values . . . . . . . . . . . . . . . . . 107

6.5 Drinfeld Integrals . . . . . . . . . . . . . . . . . . 119

6.6 Exercises ..................... 126

II Theory of Modular Forms of Several Variables 129

7 Theory of Modular Forms of Several Variables 131

7.1 The Generalized Upper Half-Plane . . . . . . . . . . . . . . 131

7.2 The Real Symplectic Group . . . . . . . . . . . . . . . . . 133

7.3 The Action of the Symplectic Group . . . . . . . . . . . . . 136

7.4 The Generalized Disc . . . . . . . . . . . . . . . . . . 140

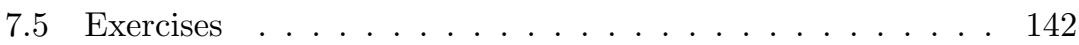

8 The Full Modular Group $\quad \mathbf{1 4 5}$

8.1 The Full Modular Group and Its Subgroups . . . . . . . . . 145

8.2 Regular Elliptic Elements . . . . . . . . . . . . . . . 148 
8.3 Siegel Modular Forms ． . . . . . . . . . . . . . . . . 150

8.4 Exercises . . . . . . . . . . . . . . . . . . 152

9 The Fourier Coefficients of Eisenstein Series 155

9.1 A Transformation Formula . . . . . . . . . . . . . . 155

9.2 Siegel's Main Formula and Its Applications . . . . . . . . . 160

9.3 Theta Series . . . . . . . . . . . . . . . . . . . . . . . . . 162

9.4 An Example to Siegel's Main Theorem . . . . . . . . . . . . 164

9.5 Exercises . . . . . . . . . . . . . . . . . . 168

10 Theory of Jacobi Forms 171

10.1 Jacobi Forms over Real Numbers . . . . . . . . . . . . . . . 171

10.2 Cayley Numbers . . . . . . . . . . . . . . . . . . . . . 174

10.3 Jacobi Forms over Cayley Numbers . . . . . . . . . . . . . 177

10.4 Jacobi Forms as Vector-Valued Modular Forms . . . . . . . 179

10.5 Examples of Jacobi Forms . . . . . . . . . . . . . . 183

10.6 Exercises . . . . . . . . . . . . . . . . 187

11 Hecke Operators and Jacobi Forms 189

11.1 Hecke Operators on Jacobi Forms . . . . . . . . . . . . . . . 189

11.2 Maaß Space . . . . . . . . . . . . . . . . . . . . . 192

11.3 Selberg Trace Formula . . . . . . . . . . . . . . . . . 195

11.4 Exercises . . . . . . . . . . . . . . . . . 197

12 Singular Modular Forms on the Exceptional Domain 199

12.1 Modular Forms on the Exceptional Domain . . . . . . . . . 199

12.2 Jacobi Forms of Degree Two over Cayley Numbers . . . . . 203

12.3 Jacobi-Eisenstein Series _ . . . . . . . . . . . . . 206

12.4 Applications to Singular Modular Forms . . . . . . . . . 208

12.5 Jacobi Cusp Forms . . . . . . . . . . . . . . . . . . . . 212

A The $p$-Adic Numbers $\quad 219$

A.1 The $p$-Adic Integers . . . . . . . . . . . . . . . . . . 219

A.2 The $p$-Adic Valuation . . . . . . . . . . . . . . 222

A.3 The Field of $p$-Adic Numbers . . . . . . . . . . . . 226

A.4 Kummer's Congruences . . . . . . . . . . . . . . . . 228 
B Weighted Sum Formulae of Multiple Zeta Values 235

B.1 Introduction and Notations . . . . . . . . . . . 235

B.2 The Proof of Theorem B.1.1 and Its Consequences . . . . . 238

B.3 The Proof of Theorem B.1.3 and Generalizations . . . . . . 242

B.4 Generalizations . . . . . . . . . . . . . . . . . . 244

C The Density of a Quadratic Form on Cayley Numbers 251

C.1 Preliminaries and Notations . . . . . . . . . . . . . . 251

C.2 Interpretations as Densities . . . . . . . . . . 255

C.3 Densities at Finite Places . . . . . . . . . . . . . . . 259

C.4 Densities at Infinite Places . . . . . . . . . . . . . . 263

$\begin{array}{ll}\text { Bibliography } & 269\end{array}$

$\begin{array}{ll}\text { Index } & 275\end{array}$ 